\title{
Thematic course design for an undergraduate photonics engineering course
}

\section{Barry Shoop}

Barry L. Shoop, "Thematic course design for an undergraduate photonics engineering course," Proc. SPIE 9666, 11th Education and Training in Optics and Photonics Conference, 966614 (5 June 2009); doi: 10.1117/12.2207967

Event: Eleventh International Topical Meeting on Education and Training in Optics and Photonics, 2009, St. Asaph, United Kingdom 


\title{
Thematic course design for an undergraduate photonics engineering course
}

\author{
Invited Paper \\ Barry L. Shoop \\ Department of Electrical Engineering and Computer Science and Photonics Research Center \\ United States Military Academy, West Point, New York 10996 \\ Barry.Shoop@usma.edu
}

\begin{abstract}
The traditional approach to undergraduate engineering course design is to first present underlying theoretical concepts in the course curriculum and then subsequently apply these theoretical concepts to system-level applications. A traditional photonics engineering course, for example, first reviews electromagnetic field theory, addressing essential concepts from geometrical and wave optics followed by an investigation of the interaction of photons with materials. Building upon these fundamental principles, the students then study the operating principles and design considerations of photoemitters, photodetectors, optical waveguides, and optical modulators. Individual devices are then combined in the design, construction and testing of a system - an example being a fiber optic communication link. This approach is often frustrating for the students because it is the applications that motivated them to study the subject and in many cases they have lost focus and interest well-before the applications are covered. This challenge can be overcome by deliberate course design where relevant thematic applications are introduced early in the course and routinely revisited as a referent. This approach has been shown to effectively motivate student-centric, inquiry-based learning.

This thematic course design framework was applied to an undergraduate photonics engineering course, ${ }^{1,2}$ at the U.S. Military Academy at West Point where an emphasis was placed on inquiry-based investigation of a wavelength division multiplexing communication system introduced during the first lesson of the course and subsequently revisited throughout the remainder of the course. The underlying theory necessary to understand foundational concepts, device behavior and subsystem operation was presented in a just-in-time fashion.
\end{abstract}

Key Words: photonics engineering, thematic course design, inquiry-based learning.

\section{INTRODUCTION}

Many undergraduate courses in engineering programs are structured to present underlying theoretical concepts first in the course curriculum and then subsequently, after covering the necessary theoretical constructs, introduce system-level applications. A traditional photonics engineering course, for example, first reviews electromagnetic field theory, addressing essential concepts from geometrical and wave optics followed by an investigation of the interaction of photons with materials. Building upon these fundamental principles, the students then study the operating principles and design considerations of photoemitters, photodetectors, optical waveguides, and optical modulators. Individual devices are then combined in the design, construction and testing of a system - an example being a fiber optic communication link.

Additionally, concept reinforcement within individual courses and among courses in the curriculum is frequently not deliberate. Students often fail to understand the importance of linkages within and among courses and subjects, and instead view their education as a series of disjoint and unrelated topics and courses. Making conceptual linkages and transferring knowledge from one context to another is a particularly important skill to teach students and, as importantly, is an effective teaching technique. Learning new information is more effective and efficient if the new information is framed within a known context and in fact, deduced from an established knowledge base. Many of the most accomplished and 
successful scientists and engineers understand new concepts by relating them to foundational theories and framing the new concepts within the context of their own knowledge and experience. In a course like photonics engineering, earlier courses in chemistry, physics, electromagnetic fields and waves, signals and systems, and solid state electronics must all be leveraged to make the most effective use of time, extend previously developed foundational concepts to new applications, and deliberately reinforce those concepts.

Both of these challenges can be overcome by deliberate course and curriculum design. The first can be overcome by designing courses around a system-based application and the second by deliberate integration of the curriculum. The system-based application can be introduced early in the course and used effectively to motivate student-centric, inquiry-based education. Integration of the curriculum begins by identifying common foundational themes within and between courses, and highlighting these to students as the topical coverage warrants. Deliberate integration of the curriculum is accomplished by not only identifying the foundational themes through conceptual abstraction, but also, by design of common exemplars. We have begun deliberate curriculum design where topical linkages and recurring thematic examples are used to demonstrate course-to-course disciplinary linkages and reinforce foundational concepts. Curriculum integration will not be addressed in this paper. However, one example of a deliberate curriculum integration theme which we have developed strives to unify the development of topics such as resonance, filtering, stability, transmission line behavior, and spectral characteristics of lasers in courses such as signals and systems, basic electric circuits, controls, electromagnetic fields, and photonics from mathematical models and analysis techniques associated with second-order linear system response describing damped harmonic oscillators. ${ }^{3}$

This paper first introduces the original course design for an upper-division, undergraduate photonics engineering course and then describes the restructured course and the assessment of student results across the two different course designs.

\section{ORIGINAL COURSE CONSTRUCT}

Photonics Engineering is an upper-division undergraduate course taken by electrical engineering majors at West Point. The course began as a traditional introductory photonics engineering elective course in the electrical engineering curriculum. The course syllabus from academic year 2001 is shown in Figure 1. The text used for this course was and continues to be Fundamentals of Photonics by B. E. A. Saleh and M. C. Teich. This course followed the traditional approach of theory - devices - applications. In the beginning of the course theoretical contructs were covered including a review of linear system theory, electromagnetic fields and waves and some circuit analysis and electronics. This was followed by geometrical optics, wave optics, electromagnetic optics and an introduction to Fourier optics. Once this foundational material was covered, devices were introduced including lasers and light emitting diodes, modulators, photodetectors, and optical waveguides. At this point in the course, the students were able to synthesize the previous course material and address systems including fiber optic communications, imaging, and image processing applications. The early laboratories in this course were independent, self-contained laboratory exercises focused on verification of fundamental physical principles similar to those found in a traditional physics course that covers similar material. Since the geometrical optics and polarization laboratory was the first physical optics laboratory in the course, the objectives were twofold. First, students were to become familiar with basic optics laboratory hardware and laser safety procedures. Secondly, they were to become familiar with lenses, waveplates, linear polarizers and simple optical systems and verify the predictions of geometrical optics. The purpose of the diffraction and interference laboratory was to become familiar with diffraction gratings, pinholes, and optical slits and verify the predictions of optical diffraction and interference. Laboratory VI on lesson 36 of a 40 lesson course was the first time the students began assembling subsystems into a functional application of a fiber optic communication system. 


\begin{tabular}{|c|c|c|}
\hline Lesson & Topic & Assignment \\
\hline 1 & Introduction \& Review of Electromagnetic Wave Theory & pp. 43-49, 158-167 \\
\hline \multicolumn{3}{|c|}{ Geometrical Optics } \\
\hline 2 & Geometrical Optics: Snell's Law and Matrix Methods & pp. $6-15,25-26$ \\
\hline 3 & Geometrical Optics: The Eikonal Equation, Optical Components & pp. $26-31$ \\
\hline \multicolumn{3}{|c|}{ Electromagnetic Fields \& Waves } \\
\hline 4 & Eectromagnetic Waves: Plane Waves, Absorption, and Dispersion & pp. 167-182 \\
\hline $5 \mathrm{Lab}$ & Geometrical Optics \& Polarization & \\
\hline 6 & Power Flow and Polarization & pp. 195-203 \\
\hline 7 & Pdarization Continued, Anisotropic Media, Reflection \& Refraction & pp. 203-214, 230-234 \\
\hline 8 & Fresnel Equations, Critical Angle, Brewster Angle, Phase Shifts & pp. $203-214,230-234$ \\
\hline 9 & Diffraction \& Interference & pp. $60-70$ \\
\hline 10 & Fourier Optics \& Holography & pp. $143-151$ \\
\hline $11 \mathrm{Lab} \|$ & Diffraction \& Interference & \\
\hline 12 & Gaussian Beams I: A Solution to the Wave Equation & pp. $81-92$ \\
\hline 13 & Gaussian Beams II: Matrix Methods & pp. $92-100$ \\
\hline \multicolumn{3}{|c|}{ Resonators and Lasers } \\
\hline 14 & Optical Resonators I: Fabry-Perot \& Spherical Mirror Resonators & pp. $311-321,327-336$ \\
\hline 15 & Optical Resonators II & pp. $311-321,327-336$ \\
\hline 16 & Review & Lessons 1-13 \\
\hline 17 & Midterm Exam I & Lessons 1-13 \\
\hline $18 \mathrm{Lab}$ III & Holography & \\
\hline 19 & Lasers I: An Optical Oscillator; Energy Levels & pp. $495-496,424-432$ \\
\hline 20 & Lasers II: Photon-Atom Interactions: Transition Rates \& Lineshape & pp. $432-449$ \\
\hline 21 & Lasers III: Laser Amplifiers: Gain \& Gain Saturation & pp. 461-476 \\
\hline 22 & Lasers IV: Laser Output Characteristics & pp. $496-515$ \\
\hline 23 & Lasers V: Pulsed Operation: CW, Q Switching, and Mode Locking & pp. 515-524 \\
\hline 24 & Photons in Semiconductors: Interaction of Photons \& Charge Carriers & pp. $543-569$ \\
\hline 25 & Light Emitting Diodes \& Semiconductor Lasers & pp. 593-632 \\
\hline 26 Lab IV & LED + LED Circuit and Laser Diodes & pp. $800-809,812-815$ \\
\hline 27 & Photodetectors: Properties \& Photoconductivity & pp. $645-657$ \\
\hline 28 & Review & Lessons $13-26$ \\
\hline 29 & Midterm Exam II & Lessons $13-26$ \\
\hline \multicolumn{3}{|c|}{ Modulation \& Guided Waves } \\
\hline 30 & Modulation I: Acousto-Optic Effect & pp. $800-809,812-815$ \\
\hline 31 & Modulation II: Acousto-Optic Devices & pp. $815-825$ \\
\hline 32 & Guided Waves I: Optical Waveguides; 1D Planar Guide & pp. $238-258$ \\
\hline 33 Lab V & Acousto-Optic Modulation & \\
\hline 34 & Guided Waves II: Modes, Step \& Graded Index Fibers & pp. 273-296 \\
\hline 35 & Guided Waves III: Attenuation and Dispersion & pp. $296-306$ \\
\hline $36 \mathrm{Lab}$ Vl & Fiber Optic Communication Link I & \\
\hline \multicolumn{3}{|c|}{ Special Topics in Photonics } \\
\hline 37 & Special Topics in Photonics Engineering & Handout \\
\hline 38 & Holography & Handout \\
\hline $38 \mathrm{Lab}$ VII & Fiber Optic Communication Link II & \\
\hline 39 & Special Topics in Photonics Engineering & Handout \\
\hline 40 & Course Review & \\
\hline
\end{tabular}

Figure 1. Photonics engineering course syllabus for academic year 2001.

\section{RESTRUCTURED THEMATIC COURSE DESIGN}

In academic year 2004 we deliberately restructured our photonics engineering course to integrate a thematic application approach. We also moved from a traditional lecture-style approach to an inquirybased approach to student-centered learning. This course restructuring required a substantial restructuring of the course syllabus to accommodate a logical approach to inquiry-based learning. The restructured course syllabus for academic year 2006 is shown in Figure 2. The course text remained Fundamentals of Photonics by B. E. A. Saleh and M. C. Teich. One could argue that the traditional approach to course design can be described mathematically as

$$
\text { Theory } \rightarrow \quad \text { Devices } \rightarrow \quad \text { Systems . }
$$

In contrast, this new approach can be described as

$$
\text { Applications(Devices(Theory)) . }
$$


After considering the topical coverage of the course and relevant disciplinary applications, this rationale led us to develop an optical communication system-based thematic application, shown in Figure 3 . Here, an emphasis is placed on an inquiry-based investigation of an optical communication system which is introduced during the first lesson of the course and subsequently used as the educational vehicle throughout the remainder of the course. In keeping with the rationale of Equation (2), the underlying theory necessary to understand foundational concepts, device behavior and subsystem operation is presented in a just-in-time fashion. The goal of this methodology is to motivate student interest and learning throughout the course and develop inquiry-based techniques.

\begin{tabular}{|c|c|c|}
\hline LeSSON & Topic & Assignment \\
\hline 1 & 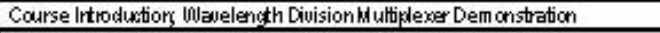 & None \\
\hline \multicolumn{3}{|c|}{ Transmitter: Optical Sources } \\
\hline 2 & Lasers l: An Opatical Oscilletor, Enerci Levek, BoltemanDistribution & $40.495-495,424428,482-433$ \\
\hline 3 & Optical Resorators l: Planar klimor \& Fakry-Pend Resorators & pa 311320 \\
\hline 4 & Lasers II: Photon-Btom Interactions, Transition Rates and Lineshape & pan 434445 \\
\hline 5 & Lasers III: Laser inmplifiers: Lineshzo kroadenimg \& Am plifier Charasteristics & pan 444449 \\
\hline 5 & Gaussian Beam s l: Solution to lólave Equation & w. 81.92 \\
\hline 7 & Optical Resorators II: Spherical Wirrors \& Gaus san Wh odes & $\mathrm{pan} 327336$ \\
\hline 8 & Lasers lu: Laser Amplfiers, Las er Oscilation & pap. $451-475,495-503$ \\
\hline 9 & Class Drop & \\
\hline 10 & Lasers u. Laser output Characteridics, Lak familaríation & pas 503515 \\
\hline \multicolumn{3}{|c|}{ Transmitter: Optical Modulation } \\
\hline 11 & Electromagnetis lolves: Wedium interactions & Pa. 158-174 \\
\hline Labl & Fiber Optics Vicice and DataKit & \\
\hline 12 & Moduators l: Bragg Dffration & pax $800-808,812-814$ \\
\hline 13 & Woduators II: Asousto-optic Effect & pas 815825 \\
\hline \multicolumn{3}{|c|}{ Transmittor: Optical Multiplexing } \\
\hline 14 & Geom etrical Optics I: Reflection and Refration, Watrix Wethocts & pap 2-15 \\
\hline 15 & Geom etrical Optics II: Imaging & 10.2531 \\
\hline Labl| & Moduation & \\
\hline 16 & Wiktterm Exam I & Lessors 1-13 \\
\hline 17 & Gaussian Beam s II: Trars mission through Optical Components & $\$ 2.92-100$ \\
\hline 18 & Polarization l: Fresnel Equations & pap 194197, 203209 \\
\hline 19 & Polarization ll: Crtical Angle Brewster's Angle & pap 203209 \\
\hline \multicolumn{3}{|c|}{ Transmission Channel: Optica Waveguide } \\
\hline 20 & Class Drop & \\
\hline Lab III & Geometrical Optics & \\
\hline 21 & Guided linaves I: Planar Wiror lôlaveguides & pa 238.248 \\
\hline 22 & Class Drop & \\
\hline 23 & Guided lobues ll: Step-IndexFibers & aㅡ 273287 \\
\hline 24 & Guided lontaves III: Atterwation \& Dispersion & pap. 296306 \\
\hline \multicolumn{3}{|c|}{ Receng: Optical Demultiplexing } \\
\hline & Fiekl Trip -P hotorics Research Center & \\
\hline 25 & Diffrastion ard linterfererse & $190.60-70$ \\
\hline \multicolumn{3}{|c|}{ Receiner: Optical Detector } \\
\hline 25 & Phatons in Sem iconductors: Interaction of Photons and Charge Cariers & pa 543-560 [Review] \\
\hline 27 & Photons in Sem iconductors: Diodes & \\
\hline 28 & Photodedectors l: Properties, Photoconductivity & 90.645657 \\
\hline Lably & Pdanization \& Fiber Optics & \\
\hline 20 & Photodetentors II: Photodiodes & pap. 657661 \\
\hline 30 & Design Prgiect O verview, (0IPR II Revien & \\
\hline 31 & Wiktterm Exam II & Lessons 1429 \\
\hline \multicolumn{3}{|c|}{ Adranced Topics in Photonics } \\
\hline 32 & LEDs & Pas 596609 \\
\hline Labu & Lasers and LEDs & \\
\hline 33 & Semisonductor Lasers & 로 609631 \\
\hline 34 & Class Drox Desian Project & \\
\hline 35 & Class Dropx Design Project & \\
\hline 35 & Class Dropx Design Project & \\
\hline $3 T$ & Fourier Optics & Handout \\
\hline 3 & Class Dropx Design Project & \\
\hline Libul & Desicn Project & \\
\hline 39 & Class Dropx Design Project & \\
\hline 40 & Design Project Presertation & \\
\hline
\end{tabular}

Figure 2. Photonics engineering course syllabus for academic year 2006.

An interesting and effective addition to this restructured course was the addition of a fiber optics voice and data kit as Laboratory $\mathrm{I}^{4}$. In this laboratory, students build a self-contained fiber optics kit. At this stage in the course they have not covered the necessary course material to fully-understand the 
components or subsystems. The purpose is to provide yet another example of a more compact, albeit simpler, commercially packaged optical communication system. At the end of the course, the students then revisit this project and write a short report that is intended to synthesize the course material and give them the opportunity to then describe all of the components and subsystems in this kit according to the knowledge they acquired in the course.

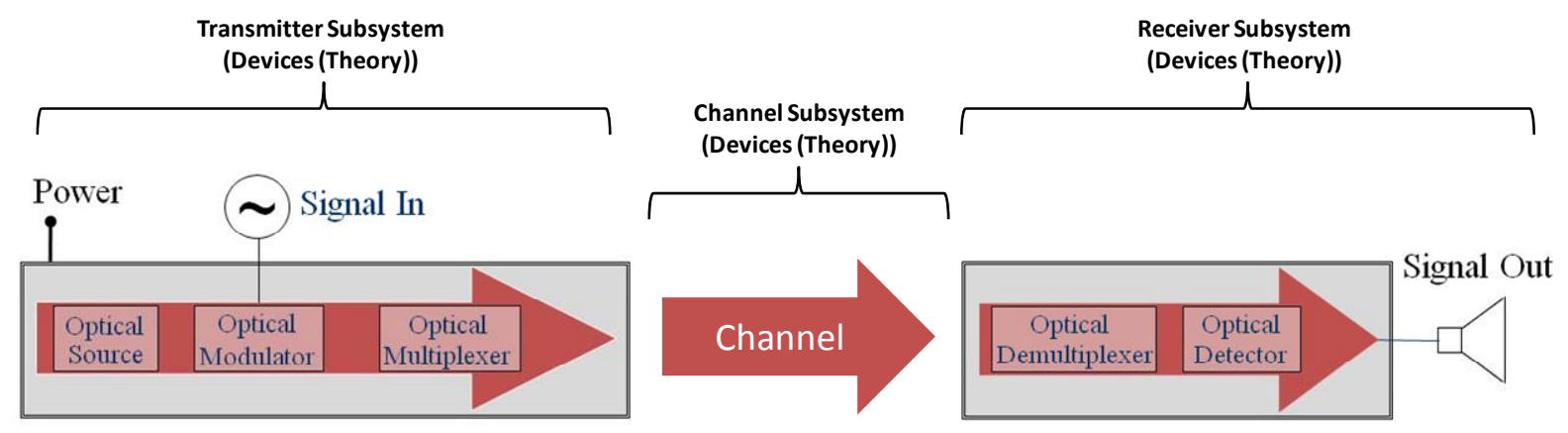

Figure 3. Photonics engineering course construct.

The course was structured to progress through the transmitter subsystem and address optical sources, optical modulation, and optical multiplexing in sequence followed by coverage of the channel subsystem and finally the receiver subsystem accounting for optical demultiplexing and optical detection.

The optical communication system used as the educational vehicle is a fully-functional wavelength division multiplexing (WDM) optical communication system shown in Figure 4. Each of the three lasers was a different wavelength and both direct modulation and acousto-optic modulation were used in the transmitter subsystem. Multiplexing was accomplished using mirrors and beam splitters. The transmission channel subsystem was comprised of a free-space component and an optical fiber. Demultiplexing in the receiver subsystem was accomplished using a diffraction grating and optical-to-electrical conversion was accomplished using photodetectors. This WDM system was functional throughout the entire course and was brought into the classroom for the introduction of each new subsystem block of instruction to motivate the application and inquiry-based questioning. The WDM system was also available and operating during each of the laboratories.

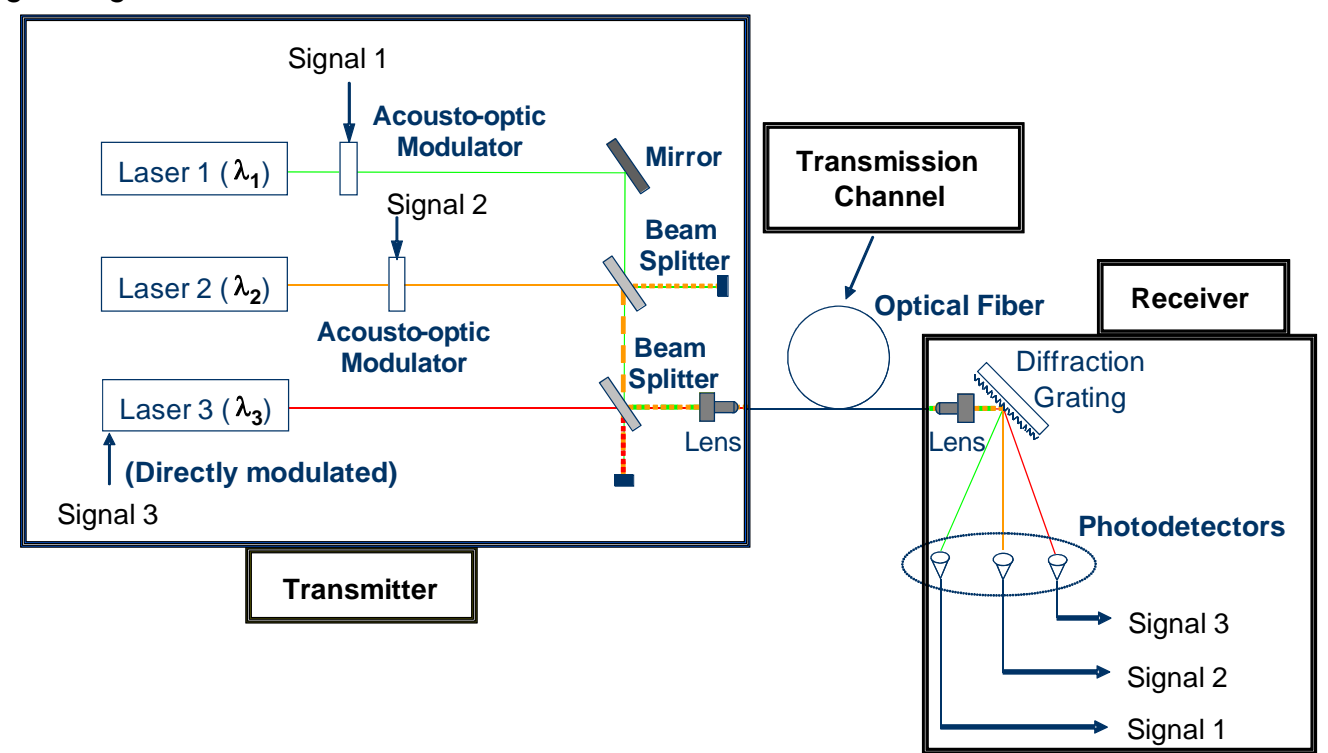

Figure 4. Photonics engineering course thematic application: wavelength division multiplexing system.

Study of a particular subsystem began by showing the students the specific functioning element or subsystem and then allowing students to ask questions about the associated devices of the operational system. Subsequent lessons were then dedicated to answering the student questions and developing the 
theory necessary to quantify or qualify the answers. An example of this inquiry-based approach is described using Figure 5 as the inquiry vehicle.

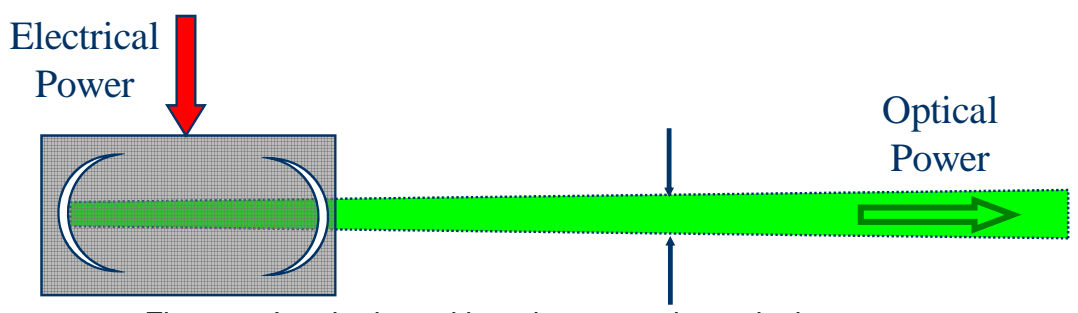

Figure 5. Inquiry-based learning example: optical source.

Student questions prompted and guided the subsequent answers and further topical investigations:
1. How do you convert electrical power into optical power?
Gain Medium
2. How is the optical power constrained in space?
3. Why does the optical power flow in a well-defined direction?
4. What properties of the optical beam can be changed?
Resonator Cavity
Electromagnetic Fields
Physical-Mathematical Analysis

To begin to answer Question 1, we considered the following:

a) Power transferred into the gain medium by a variety of energy transfer processes:

- Thermodynamic

- Ionization of atoms

- Absorption of photons

b) Gain medium properties

- Characteristic responses of constituent atoms

From these discussions we identified the necessary underlying principles and theory:

a) Principles:

- Quantized energy levels

b) Theory:

- Classical model for individual atom response

- Lineshape function (characteristic color of laser)

- Transition strength (size of "target" to absorb a photon)

- Spontaneous emission lifetime (likelihood to emit a photon)

- Density of modes

Special emphasis was placed on reinforcing concepts with device-specific laboratory exercises. Whenever possible, the linkages were established with material from previous courses. Although this approach sometimes presented difficult concepts early in the semester, requiring a level of abstraction with unanswered questions, the same material was covered in the restructured course as in the previous traditional course.

\section{ASSESSMENT.}

Based on an analysis of assessment tools over a four-year period, the academic performance of the student remained unchanged; the evaluation of knowledge and skill outcomes compared to course objectives reflected a similar level of comprehension for both the traditional and system-based instruction. Despite a reduction in course content, and sometimes incomplete theoretical development, the students were able to perform at an appropriate academic level, especially when asked to integrate photonic devices in operational systems.

Student understanding of the theoretical course concepts is easily assessed in quizzes and traditional written examinations. The linkages between the theoretical constructs, devices, and system applications are traditionally assessed in the student laboratory report submissions. We have taken additional measures to reinforce this learning component by adding several oral laboratory reports throughout the 
semester and by making the final system-level project and oral assessment. In this way the students have the opportunity to demonstrate their design project and the instructor has the opportunity to directly assess the student's understanding of the course material in the context of the design project application.

Perhaps even more important than academic performance was the increase in the student excitement for the material and the intellectual curiosity of the students. At the beginning of the semester, the average student was reluctant to ask questions or comment on the observed characteristics of the operational WDM system. By the end of the semester, the intellectual maturity of the student increased to the point that several lessons became free-flowing discussions of the topic.

\section{ACKNOWLEDGMENTS.}

This research was supported by the Army Research Office.

\section{REFERENCES}

1. G. A. Nowak, G. M. Burrow, and B. L. Shoop, "System-based Introductory Photonics Engineering Course," Invited Presentation, in Proceedings of Frontiers in Optics, Forum on Education, Rochester, NY (2004).

2. G. M. Burrow, G. A. Nowak, and B. L. Shoop, "System-based Introductory Photonics Engineering Course - II," in Proceedings of Frontiers in Optics, Forum on Education, Tucson, AZ (2005).

3. B. L. Shoop, G. A. Nowak, and L. A. Shay, "Deliberate longitudinal curricular integration: Topical linkages and concept reinforcement," Proceedings of the 2005 American Society for Engineering Education Annual Meeting, (Portland, Oregon), pp. 1-27, June 2005.

4. Model FO-30K Fiber Optics Voice and Data Kit, Elenco Electronics Inc., http://www.esssales.com/elenco/fiberoptics.html. 